\title{
A Multimodal Label-Free Imaging Study of Zeolite Crystals
}

\author{
Naomi Omori², Sara Mosca ${ }^{3}$, Ines Lezcano- Gonzalez ${ }^{2}$, Ian K. Robinson' ${ }^{2}$ Luxi Li $^{4}$, Alex G. Greenaway², \\ Paul Collier ${ }^{5}$, Andrew M. Beale ${ }^{2}$, Alessia Candeo ${ }^{1}$ \\ ${ }^{1}$ Politecnico di Milano, Piazza Leonardo da Vinci 32, I-20133 Milano, Italy \\ ${ }^{2}$ University College London, 20 Gordon Street, London, WC1H OAJ, UK \\ ${ }^{3}$ Central Laser Facility, Rutherford Appleton Laboratory, Didcot, OX11 OFA, UK \\ ${ }^{4}$ Argonne National Laboratory, 9700 South Cass Avenue, Lemont, IL 60439 (USA) \\ ${ }_{5}^{5}$ Johnson Matthey Technology Centre Blounts Court Road, Sonning Common, Reading RG4 9NH, UK \\ Email : alessia.candeo@polimi.it
}

Zeolites are complex materials that are widely employed in industry as heterogenous catalysts. Their unique open framework structures allow them to not only act as size-selective sieves, but to play host to an adsorbed phase of organic guest molecules. Imaging both the framework and the concomitant adsorbed organic material in a single micrograph is very challenging because each material has different requirements for generating image contrast. In
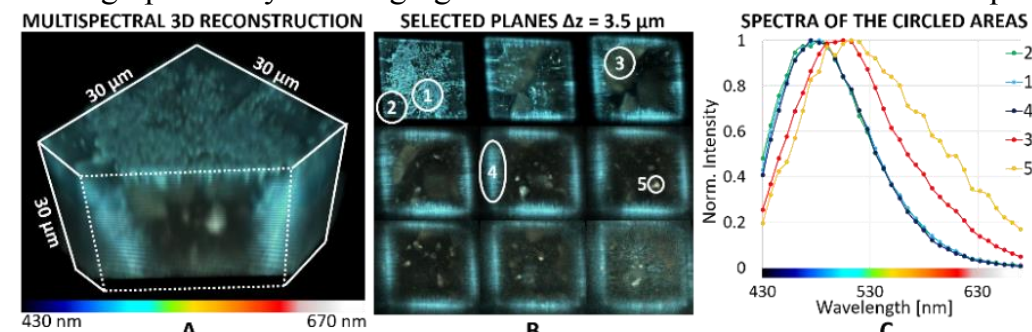

Fig. 1. 3D confocal multispectral study of a zeolite crystal. A) 3D reconstruciton of the crystal and $\mathrm{B}$ ) selected $\mathrm{z}$-planes from the stack. Colours are representative of the emission wavelength, hence colours differences indicate a different emission spectrum. C) Emission spectra correspondent to the circled areas in B.
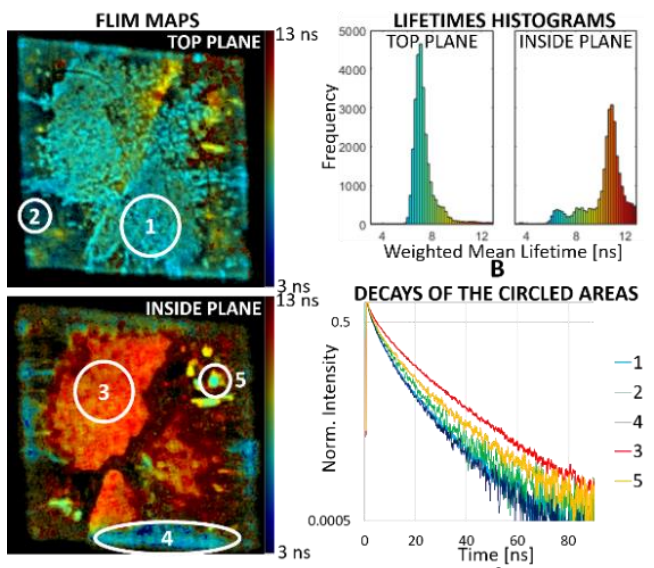

A $\mu \mathrm{m}$ chabazite crystal samples. Raman mapping is used to probe the autofluorescence of organic material generated during the detemplation process. Detemplation involves the removal of an organic templating material at the end of the zeolite synthesis process, has been previously studied using time-resolved spectroscopy [1] and is here studied in a model 30

3D confocal multispectral images (Fig. 1) acquired at $405 \mathrm{~nm}$ wavelength excitation show a striking distribution of well-defined emissive agglomerates on the surfaces of the cubic crystal facets, as well as evidence of larger sub-surface deposits distributed throughout the central volume of the crystal. The emissive species at the top and those in the inner part of the crystal differ in spectrum. The sub-surface deposits have a red shifted in emission in comparison to the surface ones, with some small agglomerates having a further broader emission spectrum. FLIM (Fig. 2) further reveals that this morphologically disparate deposits also have different lifetime behaviours. Surface deposits have a faster decay but the larger agglomerates in the

A (top) and a sub-surface one (bottom). Colours are representative of the weighted mean lifetime extracted via 3 -exponenttial fitting of the decays. B) Comparison of the lifetimes histogramsfor the two planes, with the top one showing generally a shorter range of averaged lifetimes. C) Raw decays of the areas circled in A.

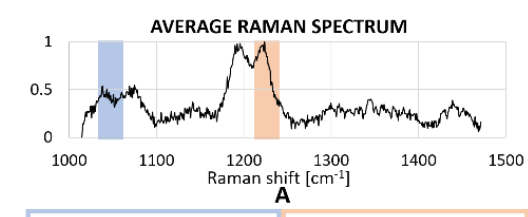
center of the facets, even though having the same emission spectrum, present a slightly longer lifetime compared to the corners. This disparity is attributed to a geometric confinement effect imposed by the host lattice on the guest molecules, which stabilizes molecular vibrations. The subsurface deposits instead have an additionally longer lifetime, with the smaller agglomerates exhibiting an intermediate lifetime. This, together with the different spectra, indicates differences in either chemical speciation or the local chemical environment. Raman mapping (Fig. 3) additionally confirms a varied distribution of bands associated with formation of polycyclic aromatic hydrocarbons stretches. Based on the morphology of deposits, spectral emission, lifetime, and Raman signature, at least three distinct emission behaviours can be identified, which could in turn be related to differences in chemical speciation [2].

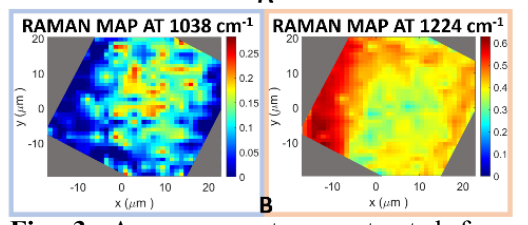

Fig. 3. Average spectrum extracted from Raman mapping. B) Selected maps correspondent to the peaks $1038 \mathrm{~cm}^{-1}$ and $1224 \mathrm{~cm}^{-1}$, assigned to template products and to the framework respectively, showing a similar heterogeneity to the other techniques. 